ENCYCLOPÉDIE Encyclopédie berbère

BERBERE

$31 \mid 2010$

31 | Matmora - Meẓrag

\title{
Mer (Vocabulaire maritime berbère)
}

\section{Serra}

\section{OpenEdition}

Journals

Édition électronique

URL : http://journals.openedition.org/encyclopedieberbere/560

DOI : $10.4000 /$ encyclopedieberbere.560

ISSN : 2262-7197

\section{Éditeur}

Peeters Publishers

\section{Édition imprimée}

Date de publication : 30 décembre 2010

Pagination : 4867-4871

ISBN : 978-90-429-2368-3

ISSN : 1015-7344

\section{Référence électronique}

L. Serra, "Mer (Vocabulaire maritime berbère) », Encyclopédie berbère [En ligne], 31 | 2010, document M90, mis en ligne le 08 octobre 2020, consulté le 12 octobre 2020. URL : http://

journals.openedition.org/encyclopedieberbere/560 ; DOI : https://doi.org/10.4000/ encyclopedieberbere.560

Ce document a été généré automatiquement le 12 octobre 2020

(c) Tous droits réservés 


\section{Mer (Vocabulaire maritime berbère)}

\section{Serra}

1 La vieille opinion qui voudrait que les indigènes de l'Afrique du Nord et tout particulièrement les Berbères ne soient en aucune façon portés vers la mer contre laquelle ils auraient une véritable répulsion se trouve en contradiction avec l'existence de groupes importants de pêcheurs berbères qui ont pratiqué et pratiquent la pêche de manière habituelle, le long de la bande côtière de l'Afrique septentrionale.

2 Ils appartiennent à des populations berbères parfois très éloignées les unes des autres, résidant, par exemple, dans le Sous, le long des côtes atlantiques du Maroc, ou à Zouara en Tripolitaine, sur les bords de la Méditerranée.

Par rapport aux zones atlantiques de l'Afrique du Nord, on a relevé les ports et le grand nombre de points de pêche des Berbères qui « en nombre variable » gagnent «leur vie en pêchant en mer » (R. Montagne 1923, p. 211).

4 Les différents aspects de cette vie maritime, les activités liées à la mer, même primitives ou traditionnelles, la technique de construction des embarcations et leur emploi à la pêche et la consommation locale du poisson trouvent leur traduction dans un vocabulaire berbère particulièrement riche.

Dans les zones méditerranéennes de l'Algérie le «simple voisinage des Européens a «fait disparaître presque complètement les populations maritimes autochtones " (Montagne, p. 173) ; en revanche, la présence de pêcheurs berbères à Djerba, en Tunisie, a été maintes fois signalée. Il y en a aussi en Tripolitaine : à Farwa où les Berbères, qui constituent presque la totalité de la population, sont tous des pêcheurs et surtout à Zouara d'où sont originaires les habitants de Farwa.

6 Ces populations ne possèdent pas des dons très prononcés en matière de navigation. Ce sont de simples pêcheurs côtiers aux techniques rudimentaires, mais ils savent en tirer un utile profit et disposent eux aussi d'un lexique maritime riche et varié qui témoigne de leur implantation ancienne au bord de la mer et d'une familiarité tout aussi ancienne avec elle.

7 L'activité maritime pratiquée le long des côtes atlantiques et méditerranéennes de l'Afrique du Nord par des Berbères et le lexique dont ils disposent rendent probable 
l'existence d'un vocabulaire maritime ancien chez les Berbères. Ce vocabulaire maritime berbère ne connaît aucune diffusion en dehors de la zone d'influence des centres berbérophones. Cela s'explique aisément en raison du faible développement des activités auxquelles il se rapporte. La pauvreté du fonds lexical berbère en termes maritimes communs à plusieurs régions connaît cependant des exceptions. Elles concernent généralement des termes qui ont été puisés dans le vocabulaire terrestre et qui ont été adaptés au langage de marine. Ainsi, par exemple, aḍu "vent»; aman, " eau »; anzar, "pluie»; azduz, "maillet pour assommer les poissons»; taferdust, «dune », etc.

8 Ce sont là des termes que l'on retrouve aussi bien sur la Méditerranée que sur l'Atlantique, comme d'autres termes qui se caractérisent par de légères variantes, tels que : tissegnit, «aiguille de mer » à Zaoura; issegni (même sens), dans le Sous ; iyyur, "quartier de lune ", à Zouara ; ayyur, « lune ", dans le Sous ; afekrun, « tortue de mer » à Zouara, lfekrun (même sens) dans le Sous.

9 Cependant les différences de vocabulaire sont plus intéressantes que les vocables communs dans la mesure où elles portent souvent sur des termes qui peuvent être fondamentaux. Ainsi, par exemple, la mer qu'on rend dans le Sous, comme dans presque toutes les zones berbères, par le terme d'origine arabe lbhar, garde à Zouara comme à Farwa l'appellation berbère ilel; ou bien rame, tolet, hameçon, appât, os de seiche, coquillage, filet, etc., auxquels font pendant dans le Sous taglut, tagust, tukt, alaf, talussi, aful, šebkt, etc., tandis qu'à Zouara, il y a respectivement lmaqdaf, skarmu, tasbennart, ta um, agrabu, abbus, aragg, etc.

Contrastant avec la nature profondément composite du point de vue lexical de la terminologie berbère concernant la mer, on trouve cependant des secteurs qui se caractérisent par leur unité.

11 Les noms de poissons, par exemple, la terminologie spécifique de l'embarcation et de ses diverses parties, le vocabulaire désignant la mer et ses différents états, les termes relatifs au régime des vents, la terminologie astronomique, sont des domaines qui présentent, en dépit des variations locales et de la variété lexicale, des caractéristiques communes.

12 Les pêcheurs berbères de la côte atlantique comme ceux de la Méditerranée utilisent par exemple pour désigner les mollusques des noms exclusivement berbères, tels que : tigri, tigrii, "moule»; aful, "toute espèce de coquillage»; amremd, "seiche", " calmar »; muntiq, "calmar commun »; abbus, " huître »; bugil, buggil, « murex commun »; taqelziwit, tagelziut, " patelle».

13 Un grand nombre de noms de poissons dans les zones berbères de l'Atlantique et de la Méditerranée est d'origine berbère mais provient du langage populaire et terrestre. Le processus par lequel le langage rural est absorbé par le langage maritime se caractérise également dans les deux zones par l'emploi de la métaphore ou de l'analogie. Certains poissons ont pour noms, par exemple, les vocables désignant la cuillère, l'aiguille, le fuseau ou d'autres objets dont ils rappellent la forme ou les caractères en fonction de leur aspect physique ou d'autres traits particuliers. On trouve pourtant: АуипјА, "grondin" (litt. la louche ou la cuiller à pot dont le poisson évoque vaguement l'image) ; tisketit, " petite tasergelt » (litt. " piquant du porc-épic », " petit fuseau à filer la laine »); agersiw, " pochetau blanc » (litt. soc); azduz n ilel, " melva » (litt. " pilon de mer »), etc. D'autres poissons sont désignés d'après la couleur qui les caractérise. $\mathrm{Ou}$ encore, lorsque le modèle est pris dans la faune terrestre, ils portent le nom de chat, de 
chien, de vache, de porc-épic, de colombe, d'hirondelle d'autres animaux dont on les rapproche par métaphore. On peut trouver pourtant les noms suivants : abrar, un très gros poisson (litt. «tacheté, bariolé ») ; auray, «mulet lippu » et «mulet-doré » (litt. «jaune »); tawayya, nom de poisson à peau noire (litt. " négresse »); tadbirt $n$ ilel, « hirondelle de mer » (litt. « rat de mer ») ; iyes n ilel, « hippocampe » (litt. « cheval de mer »); harba n ilel, mendole commune (litt. « caméléon de mer »).

Certains noms de poissons ont encore d'autres origines et semblent présenter un sens péjoratif ou caractériser un groupe. Par exemple, tudayt, " pagre, pageau rouge » (litt. "Juive ») à Zouara; appellation à laquelle font pendant dans le Sous deux noms de poissons ni berbères ni berbérisés : hut rumi, litt. " poisson du chrétien » et zebb lihudi, litt. pénis du Juif«.

Les termes qui désignent le poisson en général sont eux aussi le plus souvent berbères qu'il s'agisse d'aslem dans le Sous (asulmey chez les Touareg, asrem dans les dialectes berbères du Rif), de tigna, tegna, tegna à Zouara et à Farwa ou de trabit dans ces deux derniers endroits et dans le Djebel Nefousa.

La terminologie concernant l'embarcation, dans ses parties et ses éléments essentiels, est particulièrement berbère, mais il s'agit là aussi de termes qui proviennent du lexique terrestre de sorte que la rame est une pelle, le tolet un piquet, les couples des côtes et ainsi de suite. Les instruments nécessaires à leur réparation portent au contraire des noms empruntés à l'arabe ou à d'autres langues.

17 Le vocabulaire concernant la mer et ses états est essentiellement d'origine arabe. Le terme même désignant la mer est désormais presque partout lbahr, lbhar, lebher, d'origine arabe, à l'exception de Zouara et Farwa où le terme berbère ilel, déjà mentionné, est toujours en usage. Il existe dans le dialecte berbère de Zouara aussi des expressions pour désigner la mer calme, la mer agitée, la marée haute, qui sont d'origine berbère, à savoir respectivement : ilel yetțas (litt. "la mer dort») ou ilel d ameškun (litt. « la mer est petite »), ilel d amoqqar (litt. « la mer est grande »), ilel yeččur (litt. « la mer est pleine »).

18 L'apport de l'arabe et d'autres langues joue aussi un rôle déterminant dans le vocabulaire relatif à la technique de la navigation comme dans celui de l'astronomie où se sont conservés cependant les noms berbères fondamentaux du soleil, de la lune, de l'étoile, avec d'autres de moindre importance comme la voie lactée ou l 'arc-en-ciel.

19 On trouve encore des vocables berbères dans le langage de la pêche là où cette activité a conservé des formes anciennes, primitives ou traditionnelles. Là où elle s'est enrichie de techniques plus ou moins modernes, le lexique s'est lui aussi accru de termes empruntés.

20 La valeur technique des termes concernant tous ces secteurs est généralement fragile et floue, mais étant donné le contexte socio-économique berbère il est difficile de la déterminer et plus encore de l'évaluer par rapport aux autres vocabulaires.

21 En dépit de sa faiblesse et bien qu'il ait été contaminé par le lexique terrestre et par des emprunts aux langues étrangères, le vocabulaire maritime berbère est donc un excellent témoin des rapports que ce peuple a sporadiquement entretenu avec la mer. 


\section{BIBLIOGRAPHIE}

BRUNOT L., 1921 - La mer dans les traditions et les industries indigènes à Rabat et à Salé. Paris.

LAOUST E., 1923 - « Pêcheurs berbères du Sous », Hespéris, III, p. 237-264.

LAOUST E., 1923 - « Notes sur un vocabulaire maritime berbère », Hespéris, III, p. 297-361.

MONTAGNE R., 1923 - « Les marins indigènes de la zone française du Maroc », Hespéris, III, p. 175-215.

MONTAGNE R., 1924 - «Coutumes et légendes de la côte berbère du Maroc », Hespéris, IV, p. 111 et suiv.

MONTAGNE R., 1927 - « La pêche maritime chez les Berbères du Sud Marocain ", L'Afrique française, p. 180 et suiv.

DOLON L., 1955 - « Les poissons djerbiens », Djerba, Pêches, Histoires, Artisanat, juin 1955.

CoLIN G. S., 1924 - « Notes de dialectologie arabe. Observations sur un vocabulaire maritime berbère ", Hespéris, IV, p. 175 et suiv.

LIAUZU C., 1971-72 - « Les pêcheurs tunisiens à la veille de la deuxième guerre mondiale », IBLA, 28 , p. 295 et suiv.

LOUIS A., 1961 - « Les îles Kerkena (Tunisie) », Etude d'ethnographie tunisienne et de géographie humaine, vol. 1, Tunis.

PARADISI U., 1962 - «l pescatori berberi della penisola di Farwa (Tripolitania) », L'Universo, Rivista bimestrale dell ‘Istituto Geografico Militare, année LXII, nº 2 (mars-avril), p. 293-300.

SERRA L., 1973 - « Le vocabulaire berbère de la mer », Actes du 1er Congrès d'études des cultures méditerranéennes d'influence arabo-berbère, Alger, p. 111-120.

SERRA L., 1970 - « L'ittionimia e la terminologia marinaresca nel dialetto berbero di Zuara

(Tripolitania)», Studi Magrebini, III, IUO, Naples, p. 21-53.

INDEX

Mots-clés : Chleuh, Géographie, Lexique, Libye, Pêche 